\title{
Sources of Stimulus-Response Compatibility: Frames, Rules, and Response Tendencies
}

\author{
Barry H. Kantowitz and Dan Nathan-Roberts \\ Department of Industrial and Operations Engineering, University of Michigan, Ann Arbor, Michigan, USA
}

\begin{abstract}
While a taxonomic approach to maximizing stimulus-response (S-R) compatibility is frequently applied to improve system design, it can fall short because no taxonomy is ever complete. A model-based approach may uncover additional system flaws and can help us to understand the sources of S-R compatibility. A satisfactory model is defined as one that is acceptable on two vital dimensions: It is both theoretically correct and easy for system designers to use. This article revisits a satisfactory hierarchical model of S-R compatibility proposed by Kantowitz, Triggs and Barnes [1]. The model presupposes a recursive combination of frames, rules, and response tendencies to explain how actions are derived from stimuli mapped to responses. Several recent studies of S-R compatibility were selected to exercise the model. By using frames, rules, and response tendencies the hierarchical model generated explanations that are consistent with these empirical results. Control-display compatibility is maximized when frames, rules, and response tendencies are aligned.
\end{abstract}

\section{INTRODUCTION}

Every day we stroll through life giving relatively little thought to how we navigate through our world. All of the different systems we interact with-- elevators, stop lights, doors-- give us information about the world that we process. These systems give us stimuli-- about the floor we're on, whether we should stop or go, or where to pull or press to enter or exit a building-- that we respond to. Their inherent or learned compatibility allows us to effortlessly navigate daily life without requiring much cognitive thought. Stimulus-response (S-R) compatibility, or the degree to which a stimulus indicates or facilitates an easy response, accounts for how quickly we respond to these stimuli, and how often, or how egregious our errors are. On the spectrum of compatibility, perfectly compatible control-display mappings yield systems that are a dream to use; they're intuitive, fast, and have low error rates. On the other end of the spectrum, incompatible mappings seem frustratingly random, are hard to use, and even harder to learn. Good human factors practice seeks compatible control-display mappings.

As designers, researchers, and engineers we need a good definition of stimulus-response compatibility. We also need techniques to measure and validate S-R compatibility as a construct. Unfortunately, as previously noted by Kantowitz, Triggs, and Barnes [1], definitions can be circular, measurements can be very indirect, and construct validity can be lacking. In this article we blithely ignore such theoretical and practical tribulations in order to focus our attention on the hypothetical substrata that underlie the concept of S-R compatibility.

Compatibility affects error rate, task completion time, frustration, and potentially adoption rates of products or

\footnotetext{
*Address correspondence to this author at the Department of Industrial and Operations Engineering, University of Michigan, Ann Arbor, Michigan, USA; E-mail: barrykan@umich.edu
}

systems. A system that was perfectly compatible would allow users to have rapid error-free control, would be intuitive, and would not tax operator cognitive resources. This system would be designed in such a way that users would be able to easily and quickly control it, making no mistakes, and without requiring a lot of thought. Unfortunately, such a perfect control-display system doesn't exist for many complex tasks. Fortunately, systems that are highly incompatible don't survive for long. A very highly incompatible system would reduce the operator to belabored random guessing, or even worse, labor-intensive answers that were wrong most of the time. A very highly incompatible device would require immense amounts of thinking power to come up with frequently wrong responses, and control strategies that were also frustratingly complex and difficult to learn. As designers and researchers, it is our job to strive for maximum control-display compatibility and to understand the sources of poor compatibility.

\section{SOURCES OF COMPATIBILITY}

Compatibility is a function of how individuals process information. The same physical arrangement of controls and displays will not necessarily produce the same compatibility in all users. Operators interpret the mappings between controls and displays by using their own individual knowledge, experience, and learning. Good human factors practice considers the population of users and tries to create mappings that are consistent with how this population processes information.

Simple taxonomies of compatibilities, while widely used as design aids, can fall short because they may not fully utilize constraints imposed by the knowledge states of the population of operators. In this analysis, control-display compatibility is determined jointly by the mappings provided by the system designer and the mental states of the population of operators. Hence design of systems can be improved by utilizing models that explicitly consider operator cognition and knowledge as key factors that influence perceived S-R compatibility. 
One such model has been proposed by Kantowitz, Triggs, and Barnes [1]. As shown in Fig. (1) the model uses the constructs of frames, rules, and response tendencies to explain how stimuli elicit responses. Here we only recapitulate key aspects of this model; the interested reader is directed to the original source for additional explanation. Frames are a cognitive tool for organizing knowledge and experience. Frames place a limit on the number of likely actions and so improve compatibility by decreasing potential errors and speeding responses. Rules are mental representations of condition-action linkages that are simpler than frames. Rules can be embedded within frames. For example, a common rule for increasing the volume of a radio is to rotate its volume control clockwise. But clockwise rotation of a control does not always increase output. When the control at hand is a water faucet, clockwise rotation decreases water flow. So frames are needed to inform operators which rules will be successful. A frame related to fluid flow selects a different control rule than a frame related to electronic devices. Response tendencies are directly learned connections between stimuli and responses. Response tendencies can be invoked by cognitive structures or by less cognitive mechanisms such as association and repetition.

In this hierarchical model, frames, often triggered by the environment, control which rules are selected by the user. The frame and the rules that it activates control which response tendencies are activated. Response tendencies, in turn, control responses. The power of this model is increased by the hierarchical recursive arrangement of frames, rules, and response tendencies shown in Fig. (1). The final strength of a response tendency is the sum of excitation produced at each level of the model. A particular response will be made when the stochastic sum of: (a) direct response tendencies plus (b) rule excitation plus (c) frame excitation exceeds a response threshold. Excitation can also be negative (inhibition), making a particular response less likely to exceed the threshold. Support for the basic idea that the strength of a stereotype can be represented as the sum of several components has been provided by Hoffmann [2], who demonstrated that responses to linear displays with rotary controls can be predicted by aggregating compatibility principles. Another example of excitation summation comes from the Simon effect [3] which shows that even irrelevant spatial stimulus information activates response tendencies. In the Simon effect response strength is accumulated by the response location that corresponds to the stimulus location. In terms of the model (Fig. 1), this is explained by summating activation from two different levels, response tendencies and rules. Even though the rule --respond to the location where stimuli occur-- is inappropriate in the Simon effect, it nevertheless produces response activation. So there is ample support for the basic assumption of the model that response activation proceeds in parallel, driven by all three levels proposed by the model.

In summary, frames control rule selection, rules control selection of response tendencies, and response tendencies control responses. However, what if there is no frame, rule or response tendency information to guide response selection? We call this dismal state of knowledge Level Zero.

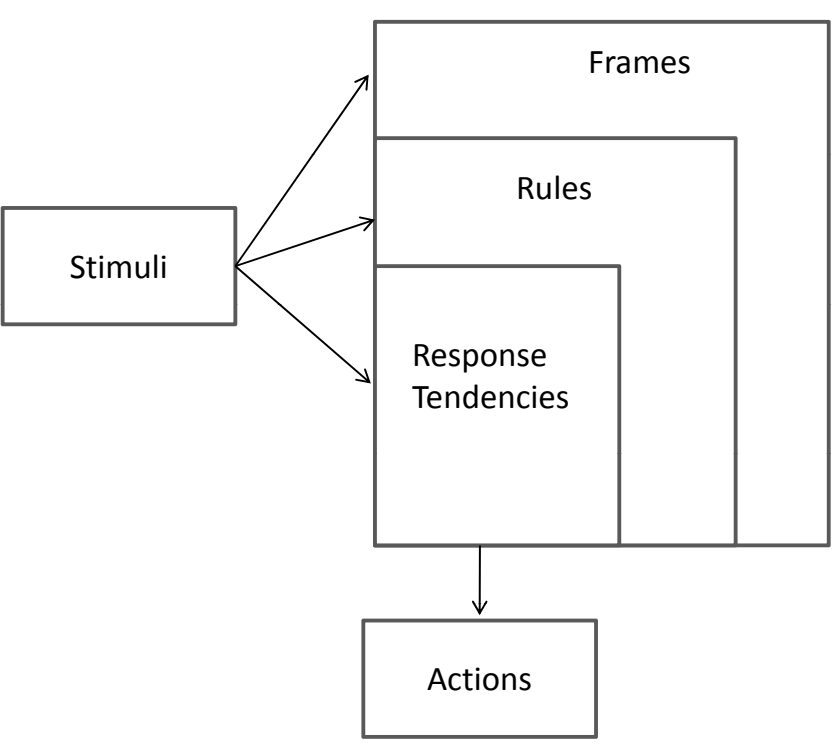

Fig. (1). Hierarchical relations between frames, rules, and response tendencies. From [1].

When response tendencies are at Level Zero, there is no connection between stimuli and responses. The connections must be learned by rote practice with feedback required for correct and incorrect responses. This situation in a controldisplay system is poor human factors practice because it fails to take advantage of any complex cognitive strategies the operator might devise. Instead, learning occurs by association and the human operator is reduced to the cognitive level of a pigeon pecking in a Skinner box. When faced with this kind of paltry information, operators will often try to invent rules to organize the response tendencies, even if the rules prove to be superstitious and only partially correlated to system mappings.

When rules are at Level Zero, operators can be quite frustrated. Even very simple rules can be most helpful. For example, what is the mapping between stimuli and responses? Is it one-to-one, many-to-one, or one-to-many? While most control-displays mappings are one-to-one, as in a choice reaction-time task where each stimulus has a unique response, the other mappings are logically possible and do occur in real systems. Many system displays have a range of values (normal operation) where no control action is required or appropriate. The operator should take action only when a threshold value is exceeded. This is similar to a Donders Creaction where one stimulus calls for a response while another does not.

One common simple rule in one-to-one mappings directly links the iconic value of a stimulus to an equivalent response icon, e.g., if the display is red press the red STOP button. The iconic values need not necessarily have a highlevel cognitive interpretation, e.g., if the display is a square press the square button and if the display is a circle press the round button. Such simple rule-based tasks can also be performed successfully by pigeons and so do not represent the pinnacle of good human factors practice unless the system being controlled is very simple with a limited number of control states such as only ON and OFF. It is difficult to 
find systems, outside the experimental psychology laboratory, where rules are at Level Zero. Most systems have been designed by engineers who at least hold implicit rules about how the system should operate. Problems arise because operators may use different rules than those intended by the designer; this occurs when designers fail to make their implicit rules explicit or have different training and experience than the typical user. Problems also arise when there is more than one way to adjust the same thing. For example, if temperature of a power plant can be reduced by opening a heat release valve, or increasing water flow, operators may not understand how different options can combine to reduce temperature in extreme situations. These confounding variables can lead to unpredicted results when operators don't fully understand their interactions on a rule level.

When frames are at Level Zero, the operator has no idea as to what set or subset of rules should be applied. All rules seem appropriate. While this might work for a very simple system, most situations call for different rules depending upon the state of the system. For example, an anesthesiologist would use one set of rules to interpret displays when a patient is first put under, another set when anesthesia is stabilized and maintained, and yet another set when the patient is returned to normal respiration. The novice operator, who has yet to establish a set of frames may well be functioning initially at Level Zero, lacking the wealth of cognitive knowledge available to the experienced operator.

As the model of compatibility is recursive, an operator functioning at Level Zero will drop to the next lowest level. For instance, if frames are at Level Zero, the operator will try to apply rules. If rules are at Level Zero the operator descends to response tendencies. If response tendencies are at Level Zero, the operator must use feedback to learn correct associations.

In examining the Frames, Rules, Response Tendencies hierarchy, we can anticipate the error types associated with various incompatibilities. Table 1 below outlines misalignments and their predicted outcomes based on Reason's Generic Error Modeling System (GEMS) [4]. The first three columns of the table represent the hierarchical model (Fig. 1). The last two columns show how misalignments of frames, rules, and response tendencies are mapped to the GEMS model.

The goal of most basic researchers is to produce a model or theory that is superior to other models. Consider two theoretical models - A and B. Model A can be better than Model B in several ways [5]. One model may predict response time in milliseconds while another might only predict ordinal relationships for different S-R mappings. If both models predict the data equally well, the model with fewer parameters is better (Occam's razor); if two theories are equally parsimonious the one that fits the data better (to say five decimal places instead of only three decimal places) is superior. We do not claim in this paper that the model espoused herein (Fig. 1) is the best model of S-R compatibility and argue only that it is a satisfactory theoretical model. Hence we do not compare this model to other theoretical compatibility models. Since this approach may appear odd, and perhaps heretical, to some basic researchers additional explanation for it is required.

We believe that system designers should use models that are theoretically correct. However, many system designers do not bother to use theoretical models at all [6] because the models produced by basic research too often are not formulated in a manner that is convenient for the practitioner who has great difficulty in determining what specific prediction is made when design A is contrasted to design B. We call this property the usability of a model. The usability of the model may depend upon the skills and software available to the system designer. For example, a production system model (e.g., [7]) might be considered extremely usable by its creators but could be utterly unusable in its original format by most system designers who lack the software and training to apply it to a design problem.

Model A can be more usable than Model B in several ways. For example, a usable model of compatibility must be tied to specific physical parameters of controls and displays such as orientation. But a usable model cannot be so specific that it only considers a very small set of control-display mappings (e.g., a digital display mapped to a rotary control located in a parallel plane.) We do not claim that the model espoused here is the most usable technique for evaluating control-display compatibility and argue only that it is sufficiently usable for most system designers. A taxonomy

Table 1. Anticipated Errors Associated with Various Compatibilities Using the Generic Error Modeling System [4]

\begin{tabular}{|c|c|c|c|c|}
\hline Frame & Rule & Response Tendency & Outcome & Effect on Data \\
\hline Aligned & Aligned & Aligned & Errors limited to system malfunction & Fast proper responses \\
\hline \multirow[t]{3}{*}{$\begin{array}{l}\text { Mis } \\
\text { aligned }\end{array}$} & & & $\begin{array}{l}\text { Mistake, misapplies a good rule, such as } \\
\text { looking the wrong way before crossing the } \\
\text { street in a foreign country }\end{array}$ & $\begin{array}{c}\text { Fast incorrect responses to a number of measures. } \\
\text { When frame is corrected, good responses come almost } \\
\text { immediately and without errors }\end{array}$ \\
\hline & $\begin{array}{c}\text { Mis } \\
\text { aligned }\end{array}$ & & Rule-based mistake, application of a bad rule & $\begin{array}{c}\text { Fast incorrect response in one measure, once } \\
\text { corrected, error free }\end{array}$ \\
\hline & & $\begin{array}{c}\text { Mis } \\
\text { Aligned }\end{array}$ & $\begin{array}{c}\text { Execution error; slip or lapse. Performing } \\
\text { intended actions incorrectly/in wrong } \\
\text { sequence, or forgetting to perform an } \\
\text { intended action }\end{array}$ & $\begin{array}{c}\text { Inconsistent results, learning effect as skills improve. } \\
\text { Often motor level errors }\end{array}$ \\
\hline \multicolumn{3}{|c|}{ Not present (level zero) } & $\begin{array}{c}\text { Mistakes and other errors in a number of } \\
\text { categories }\end{array}$ & $\begin{array}{c}\text { Incorrect and inconsistent responses, slow, or no } \\
\text { learning trend. }\end{array}$ \\
\hline
\end{tabular}


checklist is more usable but lacks theoretical content.

We believe that a better model of compatibility for system designers must satisfy both criteria and offer acceptable levels of theoretical validity and usability. A perfect theoretical model that predicts response time to the microsecond will be ignored by system designers if it is too difficult to use. Unfortunately, a procedure that is very easy to use, such as subjective rating scales, will often be used by system designers who may not know or care that it lacks theoretical validity. We define a satisfactory model as one that satisfies both criteria: It is theoretically valid and usable. A model or a design procedure that has high scores on only one dimension but fails on the other dimension is unsatisfactory.

An example of a model of S-R compatibility that we consider satisfactory has been proposed by Hoffmann [8]. It predicts response time by adding effects of only two factors: the calculated information $(\mathrm{H})$ in a response set and the calculated correlation coefficient between stimulus and response sets. It is theoretically valid because it is based on previous research and information-processing theory. It is useful because most system designers can use logarithms to the base 2 and can calculate a correlation coefficient.

We propose that the model shown in Fig. (1) is above threshold on both dimensions and thus is also a satisfactory model of S-R compatibility. Predictions of the model are consistent with the latest data. Furthermore, the constructs of frames, rules, and response tendencies can be tied to specific design parameters but are sufficiently general to apply to a wide range of problems that are of concern to system designers. These constructs also provide a theoretical richness that goes beyond the model of Hoffmann [8], thus providing the potential for our model to cover a greater scope of design problems.

\section{EXAMPLES OF S-R COMPATIBILITY}

In this section we briefly review a few empirical studies to illustrate the power of the model to help researchers categorize and understand results. Since the hierarchical model is, like Caesar's Gaul in three parts divided, we have organized the examples according to frames, rules, and response tendencies. But this is not intended to imply that only a single level is pertinent to the results. A study included under the heading Rules may very well also involve frames and a study included in the rubric Response Tendencies might also entail rules and frames.

\section{Frames}

Activating the right frame can be crucial in selecting the proper response. A proper response comes about because the correct rule is selected from the possible rules within that frame. Frames can be activated in a number of ways, such as instruction, environmental cues, association with similar frames, etc. A design that simplifies the frame choice should be the goal of the system designer. Several articles are reviewed below that highlight the importance of frames on S-R compatibility.

Bussemakers and de Haan [9]. A study with a many-toone mapping of stimuli onto responses illustrates the importance of frames. Bussemakers and de Haan [9] presented simultaneous visual and auditory stimuli. Subjects responded Yes if the picture was an animal and No if it was not. Concurrent auditory stimuli fell into three categories: (1) the sound was that produced by the animal pictured, (2) the sound was that produced by a different animal, or (3) the sound was musical and unrelated to the animal category. A fourth Silent condition had no concurrent auditory stimulus. The hierarchical model predicts facilitation when the frame activated by the sound is also activated by the picture. Since auditory stimuli (1) and (2) both activate the frame Animal there should be equal RT in both conditions and this RT should be faster than for auditory stimulus (3) where a different non-animal frame is activated. These results were obtained. The model also predicts that RT for the Silent condition should be slower than RT for auditory stimuli (1) or (2) because there is only one activation pathway for the Animal frame (visual) versus two pathways in the redundant stimuli (visual plus auditory); this prediction was supported. However, the model predicts that RT for auditory stimuli (3) should equal RT for the Silent condition since both conditions have only a single activation pathway for the Animal frame. This result was not obtained with RT for the Silent condition being greater. We suspect this outcome is associated with the greater alerting capabilities of auditory stimuli independent of how the auditory stimulus does or does not activate a cognitive frame or category. Since this experiment did not include auditory stimuli presented alone without concurrent visual stimuli, our explanation of this departure from predictions of the model cannot be evaluated from this publication.

Cho and Proctor [10]. The physical orientation of the hands and their effect on mapping up-down stimuli onto leftright movement were tested by Cho and Proctor [10]. Subjects were presented with a fixation point at the center of a screen and then an asterisk above or below the fixation point. Subjects were instructed to move a momentary toggle switch either in a right-is-up or left-is-up mapping depending on the block. The first experiment tested the effects of switch location (placing the hand to the left, center, or right of the subject's midline). This lateral eccentric placement of the switch corresponds to a frame in the hierarchical model. The instructed movement mapping is a rule. The hierarchical model predicts that for the center location the frame is not activated because there is no eccentric relationship. Thus, no competing response tendencies associated with the frame are activated and reaction time should be shortest at the body midline; this result was obtained. Results also replicated the advantage of up-right/down-left mappings with faster reaction time as found in previous studies. The hierarchical model explains this finding by invoking learned response tendencies. Increasing values are associated with movement to the right in many systems (e.g., a volume control slider increases volume when moved to the right) and increasing stimulus magnitude has been associated with upward motion of a display (e.g., a mercury thermometer). So for most user populations this mapping (up-right/down-left) has a learned advantage that yields shorter reaction times.

Chua, Weeks, Ricker, and Poon [11]. Similar to Cho and Proctor [10], Chua, Weeks, Ricker, and Poon [11] looked at body/control orientation, and its effects on compatibility. Chua et al, studied operator orientation relative to the stimulus and response; display orientation, and mapping 
rules were varied over two experiments: one that tested short discrete movements and another where subjects performed rhythmic movements synchronized with an oscillating visual display. Stimuli were positioned in front, or to the left side of subjects, and a two direction lever was positioned in front or to the right side of subjects. Subjects were given two mappings: a congruent, left-equals-left mapping, and incongruent left-equals-right mapping, and performed both reaction time and a following task in a full factorial combination of stimuli, response, and mappings.

The different combinations of stimulus and response constitute distinct frames; the mappings, as in Cho and Proctor [10], would be rules within each frame. The hierarchical model predicts worse performance for less compatible frames, and within those frames worse performance for less compatible rules. The results of the study support the model, finding higher reaction times and worse performance on the following task for different frames (e.g., stimuli in front and response control on the right of the user) and within each frame for the less compatible mapping (inverted left-equals-right mapping).

Hoffmann [2]. This study examined linear displays paired with rotary controls. Given a three-dimensional box with a vertical display, where should a designer place the rotary control? Some potential locations are (a) in the same plane as the display, (b) on top of the box, or (c) on the side of the box. Frames develop through user experience. Unless the users represent a specialized population that differs from the general public, we would expect that users have the greatest experience with co-planar displays and controls. So we as designers would not place the control in locations (b) or (c) and would expect better results for location (a). This would allow users to benefit from response tendencies associated with the co-planar frame.

Hoffmann [2] reported a perplexing result when testing two-dimensional co-planar display-control combinations. Only horizontal displays with top/bottom controls revealed significant RT decreases with increasing stereotype strength. No correlations were obtained for vertical displays which differed from the horizontal pairings by a $90^{\circ}$ rotation. Our interpretation of this result is that the student subjects in this experiment were sufficiently familiar with horizontal displays to have developed a frame for this orientation. Vertical displays, however, were less frequent for this population so that no frames were available to support common stereotypes.

Chan and Chan [12]. Another study that can be interpreted in terms of frames tested compatibility for circular displays with rotary controls. Chan and Chan [12] presented a circular display with a pointer set to one of four positions: North, South, East or West. Subjects were shown a destination mark [12] (Experiment 2) in an adjacent position that required either a clockwise or counterclockwise rotation of the control. A major goal of this study was to investigate stereotype reversibility [13]. Reversibility is an index of consistency in a population stereotype. For example, if a population rotates a knob clockwise to move a pointer clockwise and the stereotype is perfectly reversible, that population will always rotate the knob counterclockwise to effect a counterclockwise pointer movement. Although reversibility is a highly desirable property, it does not hold equally for all pointer positions. When the pointer was aimed North, the highest index of reversibility was obtained. A lower index of reversibility was found when the pointer was aimed South. Aiming the pointer East or West yielded intermediate indices of reversibility. Reversibility was also influenced by the plane in which the rotary control was positioned; locating the display and the control in the same plane maximized reversibility. We postulate that pointer position and control plane location activate frames that modulate the two control rules: rotate clockwise and rotate counterclockwise. As predicted by the hierarchical model and seen in Hoffmann [2], frames, rules, or response tendencies have an additive effect on responses. The two frames in Chan and Chan [12] showed this additive effect when both were aligned (e.g., experiment 1, plane 1, position 1).

\section{Rules}

Within frames, rules are responsible for the use of knowledge. Frames organize knowledge. As a frame is energized, the frame's strongest rules are also energized. These energized rules are then used to produce response tendencies.

Proctor and $V u$ [14]. A very recent study by Proctor and $\mathrm{Vu}$ [14] examined the effects of simple rules on S-R compatibility. In a corresponding mapping condition, stimuli were mapped to the same location as response keys: stimulus 1 corresponded to key 1 , stimulus 2 to key 2 , etc. In a mirrored mapping condition stimuli were mapped to the opposite response key: stimulus 1 corresponded to key 4, stimulus 2 to key 3, etc. The left hand was used to respond to the (temporally) first stimulus and the right hand to the second stimulus. For consistent mappings both hands were linked to stimuli by either a corresponding mapping or by a mirrored mapping. For inconsistent mappings each hand was linked by a different mapping: one hand used a corresponding mapping while the other hand used a mirrored mapping. This experimental design yielded four different within-subject conditions and the potential for various rules to be invoked.

How might a rule-based decision maker attempt to perform this sequential task? For the consistent S-R groupings one rule is sufficient for both hands. But for the inconsistent groupings two rules are necessary: one for each hand. The decision maker must first select a frame (Left Hand versus Right Hand or perhaps First Response versus Second Response since these attributes are confounded) before knowing what rule to apply. The hierarchical model predicts that some errors will occur in the inconsistent groupings when a new frame is not selected for the second response or an incorrect frame is selected for the first response. Such frame misalignment should cause a decreased RT for error responses (Table 1). Proctor and Vu [14] (Experiment 1) found that most errors in the inconsistent groupings could be attributed to what we have called misaligned frames. Unfortunately, since they did not report error RT, the speed prediction we generated cannot be evaluated from their publication. However, it would be surprising if further analysis of their data did not reveal faster error RT. 
In their next experiment Proctor and $\mathrm{Vu}$ [14] (Experiment 2) complicated the situation by creating a Mixed condition in which the Outside responses (1 and 4) used a mirrored mapping while the Inside responses ( 2 and 3) used a corresponding mapping. This mapping cannot be organized by a single rule. Instead, two rules are required. The interesting comparison here is between Consistent and Inconsistent mappings containing the Mixed condition. In the Consistent mapping with both hands using the same Mixed condition the decision maker must first select a Frame (Inside versus Outside); then the appropriate rule must be applied: mirrored for Outside or corresponding for Inside. A total of two cognitive operations are required for any response: select a Frame and apply a Rule. The Inconsistent mappings have greater cognitive complexity. First a Frame must be selected (Left Hand or Right Hand). Because one hand has a Corresponding mapping while the other hand has a Mixed mapping this is not sufficient to apply a rule in all cases. For the hand using the Corresponding mapping the corresponding rule can be applied immediately. Another level of frame selection must be performed for the Mixed condition (Inside versus Outside) before the correct rule can be applied. This adds an additional cognitive operation. Thus, the hierarchical model predicts that RT for the Inconsistent conditions should be slower than RT for the Consistent Mixed condition which in turn should be slower than RT for the Consistent Corresponding condition which requires no frame selection. Happily, this prediction is supported by Proctor \& Vu [14] (Fig. 1).

\section{Response Tendencies}

Hommel, Pratt, Colzato, and Godjin [15]. Triggered by frames and rules, or through repetition or association, response tendencies are derived from classic learning theories. Hommel, et al., [15] looked at this kind of learned association by testing the effects of directional cues on target response time when the cue direction did not predict the location of the target. Arrows and directional words are some of the first semantic elements we learn as children, or when learning a new language. They are reinforced every day as we drive, navigate shopping plazas, or even use our computer operating system. The hierarchical model predicts that when the stimulus includes directional cues, stimuli strongly linked to response tendencies, the response will be powerfully influenced. Four studies were conducted by Hommel, et al., [15], where subjects searched for a target on a screen. Each stimulus was preceded by a nonpredictive directional cue, such as the word "left," "right," "up," down," or arrows pointing in the same directions. The studies varied the stimulus onset asynchrony, response type (a letter, target detection, and target color), and in one study, even informed the subjects of the likelihood of where the target would appear. As predicted by the model, despite the experimenter's instructions, across all studies, the nonpredictive cues had an effect on response time, either decreasing response time when the cues happened to indicate the location of the target, or increasing the inhibition of return when there was a secondary peripheral cue [15] (experiment 2). For example, in experiment 3, the task was to indicate the color of a stimulus on the left or right of a fixation point by pressing a designated key; nonpredictive arrows shown $100 \mathrm{msec}$ before the stimulus, and nonpredictive words (shown $50 \mathrm{msec}$ and $450 \mathrm{msec}$ ) before the stimulus had significant effects of cue compatibility on reaction time. When the overlearned, but nonpredictive cues were presented, and were correct, reaction times were significantly faster than when they were neutral or incompatible with the actual direction of the target. Reaction times were slower when the cues were incompatible.

Ho and Spence [16]. Response tendencies are learned behaviors that often develop over years of experience. Ho and Spence, [16] tested various auditory cues for capturing driver's visual attention. Five experiments were conducted that combined, tested spatial, nonpredictive spatial, and verbal cue efficacy in alerting drivers to a car approaching quickly from the front, or quickly from the rear. The rearview mirror reflected a full size screen placed behind the car. The hierarchical model predicts that a well-practiced task like driving would become overlearned. In everyday driving, the rearview mirror is often ignored with drivers devoting far more attention to the forward view. This would predict that there is a higher response time for rare, less reinforced instances where the car is approached from the rear, versus the well-practiced car slowing or stopping responses to vehicles being followed. Ho and Spence [16] found that response times are shorter across all experiments for cues that relate to a car ahead of the study car, and slower when cues relate to a car behind them, a less practiced situation. These findings show that response tendencies are weaker when linked with stimuli with less frequent associations to driving responses.

To recapitulate a key point made at the start of this section, these findings might also be explained by rules or frames. As drivers become more experienced, they may form rules to govern the association of stimuli and responses. For example, one possible rule might be that good drivers check their rear-view mirror every fifteen seconds. With even more practice, frames may be learned. In this driving example, we might term these window frames: front window and rear window. Front-window frames more strongly energize driving responses like stepping on the brake pedal than do rear-window frames. Developmental studies using drivers with different ranges of experience would be useful to study how rules and frames emerge with increasing practice.

\section{CONCLUSION}

System designers think about S-R compatibility interminably. The usual approach to maximizing compatibility uses taxonomy (e.g. [1]), often in a checklist format. While this is a reasonable way to approach the problem, no taxonomy is ever complete. The designer might inadvertently omit some factors that could improve control-display design. Therefore, it may be helpful to also consider a more theoretical approach such as the hierarchical frames, rules, and response tendencies model discussed herein.

It seems appropriate that a recursive model be applied in a recursive manner. First the designer should try to identify relevant frames and rules. Mappings should be created so that frames and rules produce supporting, rather than competing, response tendencies. Eventually the system designer will create a nascent mapping of stimuli onto responses. Then a taxonomy-based checklist can be applied. It will most likely produce some modifications to the 
original control-display mappings. Then the hierarchical model should be applied again, to ensure that frames and rules still support compatible response tendencies. This recursive design process continues until the system designer is satisfied, or more likely, runs out of time or other resources.

Subjective ratings of compatibility using paper and pencil tests are far less accurate than objective experimental results; naïve users do not excel at predicting compatibility [8, 17]. But professional system designers should not behave like naïve users. They must rely on satisfactory models and objective data to optimize compatibility. Objective testing of designs is crucial to confirm that no inappropriate frames, rules, or response tendencies are activated, and that the best predicted designs are better than alternatives. A satisfactory model should allow designers to reduce the amount of testing needed and the model will help designers to avoid the fallacy of naïve judgments

We have tried to demonstrate in this article that the hierarchical model, although formulated two decades ago, remains valid and is consistent with the latest data. In our undoubtedly biased opinion, the model fared well. It could explain interesting features of the data. We have also generated a few new predictions of the model that can be falsified by reanalysis of existing data or by future research. But the most important ambition we hold for the model is not just that the model be correct but that it buttress a helpful way for designers to think about compatibility. To be useful, a model must be used. So we urge system designers to step back from their checklists to also consider how their design utilizes frames and rules in ways that can be planned but also might be unintended. Keep the frames and rules that yield compatible response tendencies and eschew those, especially if unintended, that generate competing response tendencies. May all your frames, rules, and response tendencies be happily aligned.

\section{REFERENCES}

[1] Kantowitz BH, Triggs TJ, Barnes VE. Stimulus-response compatibility and human factors. In: Proctor RW, Reeve RW, Eds. Stimulus-response compatibility an integrated perspective. North Holland: Elsevier Science Publishing Company 1990; pp. 465-388.
[2] Hoffmann ER. Strength component principles determining direction of turn stereotypes - linear displays with rotary controls. Ergonomics 1997; 40(2): 199-222.

[3] Simon JR. The effects of an irrelevant directional cue in human information process. In: Proctor RW, Reeve RW, Eds. Stimulusresponse compatibility an integrated perspective. North Holland: Elsevier Science Publishing Company; 1990; pp. 31-86.

[4] Reason JT. Generic Error-Modeling System (GEMS): A cognitive framework for locating human error forms. In: Rasmussen J, Duncan K, Leplat J, Eds. New technology and human error. London: Wiley 1987.

[5] Kantowitz BH, Roediger H, Elmes D. Experimental psychology $9^{\text {th }}$ ed. Belmont, CA: Wadsworth 2009.

[6] Kantowitz BH. Can cognitive theory guide human factors measurement? In: Proceedings of the Human Factors Society, 34th annual meeting. Santa Monica, CA 1990; pp. 1258-62.

[7] John, BE, Newell A. Towards an engineering model of stimulusresponse compatibility. In Proctor RW, Reeve RW, Eds. Stimulusresponse compatibility an integrated perspective. North Holland: Elsevier Science Publishing Company; 1990; pp. 427-61.

[8] Hoffmann ER. Naïve judgments of stimulus-response compatibility. 2009 unpublished

[9] Bussmakers MP, de Haan A. When it sounds like a duck and looks like a dog. Auditory icons vs earcons in multimedia environments In: Proceedings of international conference on auditory displays; 2000; Atlanta: International community for auditory display 2000; pp. 184-9.

[10] Cho Y, Proctor R. Influences of hand posture and hand position on compatibility effects for up-down stimuli mapped to left-righ responses: Evidence for a hand referent hypothesis. Percept Psychophys 2002; 64(8): 1301-15.

[11] Chua R, Weeks DJ, Ricker KL, Poon P. Influence of operator orientation on relative organizational mapping and spatial compatibility. Ergonomics 2001; 44(8): 751-65.

[12] Chan WH, Chan AHS. Movement compatibility for rotary control and circular display-computer simulated test and real hardware test. Appl Ergon 2003; 34(1): 61-71.

[13] Hoffmann ER, Brown C, Morgan S. Stereotypes for operation of water taps. In: 28th Annual Conference on Ergonomics Society of Australia. Melbourne 1992; pp. 63-72.

[14] Proctor RW, Vu K-PL. Determinants of benefit for consistent stimulus-response mappings in dual-task performance of fourchoice tasks. Atten Percept Psychophys 2009; 71(4): 734-56.

[15] Hommel B, Pratt J, Colzato L, Godijin R. Symbolic control of visual attention. Psychol Sci 2001; 12(5): 360-5.

[16] Ho C, Spence C. Assessing the effectiveness of various auditory cues in capturing a driver's visual attention. J Exp Psychol 2005; 11(3): 147-57.

[17] Payne S. Naive judgments of stimulus-response compatibility. Hum Factors 1995; 37(3): 495-506. 